\title{
Approximate Reasoning in Fuzzy Resolution
}

\author{
Banibrata Mondal, Swapan Raha \\ Department of Mathematics, Visva-Bharati University, Santiniketan, West Bengal, India \\ Email: mbanibrata@gmail.com, swapan.raha@visva-bharati.ac.in
}

Received January 2, 2013; revised February 3, 2013; accepted February 20, 2013

Copyright (C) 2013 Banibrata Mondal, Swapan Raha. This is an open access article distributed under the Creative Commons Attribution License, which permits unrestricted use, distribution, and reproduction in any medium, provided the original work is properly cited.

\begin{abstract}
Resolution is an useful tool for mechanical theorem proving in modelling the refutation proof procedure, which is mostly used in constructing a "proof" of a "theorem". An attempt is made to utilize approximate reasoning methodology in fuzzy resolution. Approximate reasoning is a methodology which can deduce a specific information from general knowledge and specific observation. It is dependent on the form of general knowledge and the corresponding deductive mechanism. In ordinary approximate reasoning, we derive $B^{\prime}$ from $A \rightarrow B$ and $A^{\prime}$ by some mechanism. In inverse approximate reasoning, we conclude $A^{\prime}$ from $A \rightarrow B$ and $B^{\prime}$ using an altogether different mechanism. An important observation is that similarity is inherent in fuzzy set theory. In approximate reasoning methodology-similarity relation is used in fuzzification while, similarity measure is used in fuzzy inference mechanism. This research proposes that similarity based approximate reasoning-modelling generalised modus ponens/generalised modus tollens-can be used to derive a resolution-like inference pattern in fuzzy logic. The proposal is well-illustrated with artificial examples.
\end{abstract}

Keywords: Approximate Reasoning; Similarity Index; Similarity Based Reasoning; Resolution Principle

\section{Introduction}

In automated theorem proving, resolution is a rule of inference leading to a refutation theorem-proving technique. Applying the resolution rule in a suitable way, it is possible to check whether a propositional formula is satisfiable and construct a proof that a first-order formula is satisfiable/unsatisfiable. In 1965, J. A. Robinson [1] introduced the resolution principle for first-order logic. A resolvent of two clauses $C_{1}, C_{2}$ containing the complementary literals $p$ and $\neg p$ respectively, is defined as $\operatorname{res}\left(C_{1}, C_{2}\right)=\left\{C_{1}-\{p\}\right\} \cup\left\{C_{2}-\{\neg p\}\right\}$,

$U$ is understood as the disjunction of the literals present in them. It is also a logical consequence of $C_{1} \wedge C_{2}$. A resolution deduction of a clause $C$ from a set $S$ of clauses is a finite sequence of clauses $C_{1}, C_{2}, \cdots, C_{n}=C$ such that, each $C_{i}$ is either a member of $S$ or is a resolvent of two clauses taken from $S$. From the resolution principle in propositional logic we deduce that, if $S$ is true under some truth valuation $v$, then $v\left(C_{i}\right)=$ TRUE for all $i$, and in particular, $v(C)=$ TRUE [2].

Example 1: Here, is a derivation of a clause from a set of clauses presented by means of a resolution Tree in Figure 1.

In first order logic, resolution condenses the traditional syllogism of logical inference down to single rule. To

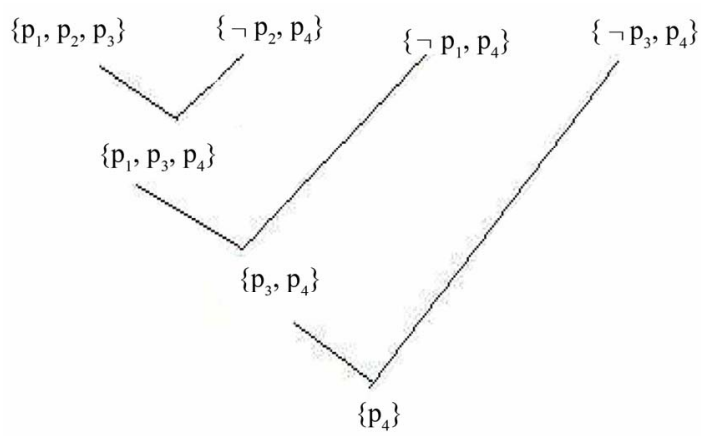

Figure 1. Resolution Tree.

A simple resoluion scheme is:

$$
\begin{aligned}
& \text { premisel }: a \vee b \\
& \begin{array}{c}
\text { premise } 2: \quad \neg b \\
\hline \text { conclusion }: a
\end{array}
\end{aligned}
$$

recast the logical inference using the resolution technique, first the formulae are represented in conjunctive normal form. In this form, all quantification becomes implicit: universal quantifiers on variables $(X, Y, \cdots)$ are simply omitted as understood, while existentially quantified variables are replaced with Skolem functions.

The first step in automated deduction in fuzzy logic 
was taken by Lee and Chang [3]. Lee's works [3,4] were continued and implemented by many researchers. Lee's fuzzy formulae are syntactically defined as classical first-order formulae, but they differ semantically as the formulae have a truth value in $[0,1]$. An interpretation $I$ is defined by an assignment $T_{I}$ of a truth value to each atomic formula, from which truth values of compounded formulae are computed [5]. Interpretation $I$ is said to satisfy (or falsify) a formula $F$, if $T_{I}(F)$, the truth value of $F$ under $I$, is at least 0.5 (or at most 0.5 ). A formula is said to be unsatisfiable if and only if, it is falsified by all its interpretations. A set $S$ of clauses is unsatisfiable in fuzzy logic if and only if, it is unsatisfiable in binary logic [3]. Mukaidono [6,7] has generalized Lee's result in the following way:

For two clauses $C_{1}, C_{2}$ in fuzzy logic, let

$C_{1}=A \vee L_{1}, C_{2}=\sim A \vee L_{2}$, where $L_{1}$ and $L_{2}$ do not contain the literal $A$ and $\sim A$ respectively as a factor and have no pair of complementary variables. Then the clause $L_{1} \vee L_{2}$ is said to be a classical resolvent of $C_{1}, C_{2}$ written as $R\left(C_{1}, C_{2}\right)$ whose keyword is $A$ and the contradictory degree of the keyword is $c d(A)$. A fuzzy resolvent of $C_{1}, C_{2}$ is written as $R\left(C_{1}, C_{2}\right)_{c d}$, where $c d=c d(A)$ is the contradictory degree of the keyword or the confidence associated with the resolvent. They have computed the truth value of $R\left(C_{1}, C_{2}\right)_{c d}$, from the truth value of $R\left(C_{1}, C_{2}\right)$ and the truth values of the atomic formulae. Then, it is proved a set $S$ of fuzzy clauses is unsatisfiable if and only if, there is a deduction of empty clause with its confidence of resolvent $c d \neq 0$ from $S$. Dubois and Prade [8] established fuzzy resolution principle in the case of uncertain proposition. In [9], antonym-based fuzzy hyper-resolution was introduced and its completeness was proved. S $a^{\prime}$ nchez et al. [10] proposed a fuzzy temporal constraint logic and introduced a valid resolution principle in order to explain/clarity some queries in this logic. Fontana and Formato [11] introduced a fuzzy resolution rule based on an extended most general unifier supplied by the extended unification algorithm. S. Raha and K. S. Ray [12] presented a generalised resolution principle that handles the inexact situation effectively and is applicable for both well-defined and undefined propositions. They associated a truth value to every proposition. We assume the fuzzy propositions to be completely true and, hence, do not associate partial truth value to the propositions. Our idea is to present, a generalised resolution principle that deals with the fuzzy propositions by the technique of inverse approximate reasoning. The advantage is that, it executes effective resolution and shows its flexibility for automated reasoning. To avoid the generic problem in handling generalised modus ponens (GMP) we use inverse approximate reasoning in fuzzy resolution. We also define fuzzy resolution on the basis of similarity/ dissimilarity measure of fuzzy sets, which is inherent in approximate reasoning.

Let us consider two clauses $C_{1}=P \vee C_{1}^{\prime}$ and $C_{2}=P^{\prime} \vee C_{2}^{\prime}$. Resolvent of $C_{1}$ and $C_{2}$ denoted by $\operatorname{res}\left(C_{1}, C_{2}\right)=C_{1}^{\prime} \vee C_{2}^{\prime}$ if and only if similarity between $\operatorname{not} P$ and $P^{\prime}$ is greater than $\epsilon$ or equivalently, dissimilarity between $P$ and $P^{\prime}$ is less than $1-\epsilon, \epsilon$ being pre-defined threshold. Instead of complementary literal, we introduce similar/dissimilar literal here. The argument form of simple Fuzzy Resolution is as follows.

$$
\begin{array}{ccc}
A \vee & B \\
\multicolumn{2}{c}{\operatorname{not} B} \\
A
\end{array}
$$

The scheme for Generalised Fuzzy Resolution is given in Table 1.

In this case, we can say that the Disjunctive Syllogism holds if $B^{\prime}$ is close to notB, $A^{\prime}$ is close to $A$.

The scheme in Inverse Approximate Reasoning looks like as given in the following Table 2.

Here, fuzzy sets $A$ and $A^{*}$ are defined over the universe of discourse $U=\left\{u_{1}, u_{2}, \cdots, u_{m}\right\}$ and fuzzy sets $B$ and $B^{*}$ are defined over the universe of discourse $V=\left\{v_{1}, v_{2}, \cdots, v_{n}\right\}$.

We shall transform the disjunction form of rule into fuzzy implication or fuzzy relation and apply the method of inverse approximate reasoning to get the required resolvent. However, in the case of complex set of clauses the method is not suitable. Hence, we investigate for another method of approximate reasoning based on similarity to get the fuzzy resolvent.

This paper consists of eight sections. In Section 2, we define and dicuss some basic concepts which are used in our paper. We briefly describe two methods of inverse approximate reasoning proposed in [13], in Section 3, and apply the method of inverse approximate reasoning towards fuzzy resolution in Section 4. Another method for fuzzy resolution in the case of complex clauses, is applied in Section 5. Section 6 is devoted with some artificial examples to illustrate the method. At last, in Section 7 some conclusions are made, follwed by some references.

Table 1. Generalised fuzzy resolution.

\begin{tabular}{cc}
\hline Rule: $X$ is $A$ or & $Y$ is $B$ \\
\hline Fact: & $Y$ is $B^{\prime}$ \\
Conclusion: $X$ is $A^{\prime}$ & \\
\hline
\end{tabular}

Table 2. Inverse approximate reasoning.

\begin{tabular}{cc}
\hline Rule (p): If $X$ is $A$ then & $Y$ is $B$ \\
\hline Fact (q): & $Y$ is $B^{*}$ \\
Conclusion: $X$ is $A^{*}$ & \\
\hline
\end{tabular}




\section{Preliminaries}

To study ordinary approximate reasoning as well as inverse approximate reasoning, we have to deal with fuzzy sets, fuzzy relations and operations on fuzzy sets, fuzzy connectives not $(\neg)$, and $(\wedge)$ and $\operatorname{or}(\vee)$. These are represented by the well known classes of negation functions (to model complement operators), continuous triangular norms (t-norms to model conjunction) and triangular conorms ( $\mathrm{t}$-conorms to model disjunction) respectively.

Some well-known t-norms and correlated t-conorms are listed in Table 3, where $M, P, B$ indicate minimum, product, bounded product and drastic product respectively for t-norms, and maximum, algebraic sum and bounded sum respectively, for the correlated t-conorms.

Typically, a fuzzy rule "If $X$ is $A$ then $Y$ is $B$ " ( $A$ and $B$ are fuzzy sets) is expressed as $I(a, b)$, where $I$ is a fuzzy implication and $a$ and $b$ are membership grades of $A$ and $B$ respectively. From an algebraic point of view, some implication operators basically identified in [14] are classified with four families $I_{i}^{T}, i \in\{1,2,3,4\}$, where $I_{1}$, known as QLimplication, is based on classical logic form

$\neg(a \wedge \neg(a \wedge b))=\neg a \vee(a \wedge b)$ and logical operators are substituted by fuzzy operators. Family $I_{2}$, often named S-implication, derives from classical logic form $a \rightarrow b=\neg a \vee b$. Families $I_{3}$ and $I_{4}$ reflect a partial ordering on propositions and are based on a generalisation of modus ponens and modus tollens, respectively. Family $I_{3}$ is known as R-implication. With reference to the t-norms and t-conorms in Table 3, the explicit expressions of fuzzy implication operators $I_{i}^{T}$ are presented in Table 4.

Table 3. t-norms and t-conorms.

\begin{tabular}{ccc}
\hline$T / T^{*}$ & $T(a, b)$ & $T^{*}(a, b)$ \\
\hline$M$ & $\min (a, b)$ & $\max (a, b)$ \\
$P$ & $a \cdot b$ & $a+b-a \cdot b$ \\
$B$ & $\max (0, a+b-1)$ & $\min (1, a+b)$ \\
\hline
\end{tabular}

Table 4. Expression of fuzzy implication operators.

\begin{tabular}{|c|c|c|c|}
\hline$I_{i}^{T}$ & $M$ & $P$ & B \\
\hline$I_{1}^{T}$ & $\max (\min (a, b), 1-a)$ & $1-a+a^{2} \cdot b$ & $\max (1-a, b)$ \\
\hline$I_{2}^{T}$ & $\max (1-a, b)$ & $1-a+a \cdot b$ & $\min (1-a+b, 1)$ \\
\hline$I_{3}^{T}$ & $\begin{array}{ll}1 \text { if } a \leq b \\
b \text { otherwise }\end{array}$ & $\begin{array}{ll}1 & \text { if } a \leq b \\
b / a & \text { otherwise }\end{array}$ & $\min (1-a+b, 1)$ \\
\hline$I_{4}^{T}$ & $\begin{array}{ll}1 \quad \text { if } a \leq b \\
1-a & \text { otherwise }\end{array}$ & $\begin{array}{ll}1 & \text { if } a \leq b \\
\frac{1-a}{1-b} & \text { otherwise }\end{array}$ & $\min (1-a+b, 1)$ \\
\hline
\end{tabular}

Here, some of the most popular implications such as the Kleene-Dienes, Reichenbach, Lukaseiwicz, Gödel and Gaines implication operators correspond to $I_{2}^{M}, I_{2}^{P}, I_{2}^{B}, I_{3}^{M}$ and $I_{3}^{P}$ respectively.

The notion of similarity plays a fundamental role in theories of knowledge and behaviour and has been dealt with extensively in psychology and philosophy. If we study the behaviour pattern of children we find that, children have a natural sense to recognize regularities in the world and to mimic the behavior of competent members of their community. Children thus make decisions on similarity matching. The similarity between two objects suggests, the degree to which properties of one may be inferred from those of the other. The measure of similarity provided, depends mostly on the perceptions of different observers. Emphasis should also be given to different members of the sets, so that no one member can influence the ultimate result. Many measures of similarity have been proposed in the existing literature $[15,16]$. A careful analysis of the different similarity measures reveals, that it is impossible to single out one particular similarity measure that works well for all purposes.

Suppose $U$ be an arbitrary finite set, and $F(U)$ be the collection of all fuzzy subsets of $U$. For $A, B \in \mathcal{F}(U)$, a similarity index between the pair $\{A, B\}$ is denoted as $S(A, B ; U)$ or simply $S(A, B)$ which can also be considered as a function $S: \mathcal{F}(U) \times \mathcal{F}(U) \rightarrow[0,1]$. In order to provide a definition for similarity index, a number of factors must be considered. We expect a similarity measure $S(A, B)$ to satisfy the following axioms:

P1. $S(B, A)=S(A, B), S(\operatorname{not} A$, not $B)=S(A, B)$, not $A$ being some negation of $A$.

P2. $0 \leq S(A, B) \leq 1$.

P3. $S(A, B)=1$ if and only if $A=B$.

P4. For two fuzzy sets $A$ and $B$, simultaneously not null, if $S(A, B)=0$ then $\min \left(\mu_{A}(u), \mu_{B}(u)\right)=0$ for all $u \in U$, i.e., $A \cap B=\Phi$.

P5. If either $A \subseteq B \subseteq C$ or $A \supseteq B \supseteq C$ then $S(A, C) \leq \min \{S(A, B), S(B, C)\}$.

A similarity measure between two fuzzy sets satisfying these axioms can also be termed as a f-near-degree. For $0 \leq \epsilon \leq 1$, if $S(A, B) \geq \epsilon$, we say that the two fuzzy sets $A$ and $B$ are $\epsilon$-similar. We now consider a definition of measure of similarity which has been proposed in $[17,18]$.

Definition 1-Similarity Indices: Let $A$ and $B$ be two fuzzy sets defined over the same universe of discourse $U$. The similarity index $S(A, B)$ of pair $\{A, B\}$ is defined by

$$
S(A, B)=1-\left(\frac{1}{n} \sum_{u}\left|\mu_{A}(u)-\mu_{B}(u)\right|^{q}\right)^{\frac{1}{q}},
$$


where $n$ is the cardinality of the universe of discourse and $q \geq 1$ is the family parameter. Here, $q$ is a real number such that a large $q$ always gives a large similarity measure. It is left to the user to set $q$ for a problem. It is easy to say that the similarity measure referred to in the Definition 1 satisfies axioms P1, P2, P3, P4 and P5.

$S(A, B) \geq S(A, C)$ implies that " $B$ is at least as close to $A$ as $C$ is to $A \% S(A, B)$ as given in Definition 1 is quite sensitive-every change in $A$ or $B$ will be reflected in $S(A, B)$. Detailed clarification of choice of the definition is described in [18].

Measure of dissimilarity is another measure of comparison of objects in literature. Many authors like B. Meunier et al. [15] have defined measure of dissimilarity in different way. However, we use the dissimilarity measure in the context of similarity measure and consider measure of dissimilarity of two fuzzy subsets $A$ and $B$ defined over $\mathcal{F}(U)$, denoted by $D(A, B)$ as $D(A, B)=1-S(A, B)$. Moreover, we assume $D(A, B)=S(\operatorname{not} A, B)=S(A, \operatorname{not} B)$. Through out the paper, we use this concept of dissimilarity. In the next section, method of inverse approximate reasoning is discussed briefly.

\section{Inverse Approximate Reasoning}

Let there be a fuzzy rule: "If $\mathrm{O}_{2}$ flow rate is LOW then heating power is LOW". Let us suppose that the "heating power is rather LOW". Then, by the method of inverse approximate reasoning with a single rule, we may construct hypotheses which would explain the causes of observation by fuzzy mathematical method. Let us consider a second example as considered in [19]. Let there be two fuzzy rules: "if the traffic is crowded then the flow is low" and "if the visibility is weak then the flow is low". Suppose, we observe "the flow is very low". According to these two linguistic rules and the corresponding observation, we first construct hypotheses by abduction such as "the traffic is very crowded" or "the visibility is very weak" or "the traffic is very crowded and the visibility is very weak". It is difficult to decide which are possible explanations for this observation. $\mathrm{N}$. Mellouli and B. Bouchon-Meunier [20] have used generalised modus ponens (GMP) to construct abductive hypotheses and used the measure of similitude to construct the best possible explanation. Here, we consider a single rule for the method of inverse approximate reasoning.

\section{Definition 2-Inverse Approximate Reasoning: Let}

$$
\text { p : If } X \text { is } A \text { then } Y \text { is } B
$$

be a given rule, where $A$ and $B$ are fuzzy subsets defined over the universes of discourse $U$ and $V$ respectively. From a given fact " $X$ is $A^{\prime}$ ", where $A^{\prime}$ is a fuzzy subset of $U$ we can conclude that " $Y$ is $B^{\prime}$ ", where $B^{\prime}$ is a fuzzy subset of $V$, by applying some method of approximate reasoning. This is called forward approximate reasoning. Now for given " $Y$ is $B^{*}$ ", we consider $\Omega\left(B^{*}\right)$ be the set of all fuzzy subsets $A^{*}$ of $U$ such that for given " $X$ is $A^{*}$ " we can conclude " $Y$ is $B^{*}$ " by the method of approximate reasoning. We have to choose the best member/s of $\Omega\left(B^{*}\right)$ (not empty) in some sense and define some inverse mapping from fuzzy subsets of $V$ into fuzzy subsets of $U$, which we refer here as inverse approximate reasoning. We shall discuss briefly two methods of inverse approximate reasoning presented in [13] to establish fuzzy resolution principle.

\subsection{Similarity Based Inverse Approximate Reasoning}

Our aim, in [13], was to feed the method of similaritybased approximate reasoning [17] to inverse approximate reasoning by writing the rule into its equivalent form. Let $X, Y$ be two linguistic variables and $U, V$ be their respective universes of discourse. Two typical propositions "p" and "q" are given in scheme as presented in Table 2 and we may derive a conclusion according to similarity based inference method [17] of the scheme in Table 2. The membership values of $A, A^{*}, B$ and $B^{*}$ are defined as before. Unlike the existing similarity based methods, a convenient way to represent a rule given by premise " $\mathbf{p}$ " is in the form of a fuzzy relation. The rule in premise "p" may be transformed into its equivalent form " $p$ " of the given premise " $\mathbf{p}$ ". We represent this equivalent rule by a fuzzy relation $R \in[0,1]^{V \times U}$ [21]. Usually, $R$ is defined on the basis of one of the operation $\theta \in\{\wedge, *, \rightarrow\}$, where $*:[0,1]^{2} \rightarrow[0,1]$ is associative, commutative and the conjunction operator in the GL-monoid $\langle[0,1], \leq, *\rangle . \rightarrow$ is the residuation operation associated with the conjunction $*$ and can be viewed as the valuation function for the implication; * is an alternative to the lattice operation $\wedge$ (in this case simply the "min" operation) for the valuation of the conjunction. Then for a given fact, the similarity between the fact and the antecedent of the equivalent form of the given rule denoted by "p" is computed and is used to modify the relation $R$. Here, every change in the concept, as it appears in the conditional equivalent premise and in the fact, is incorporated into the induced fuzzy relation (say, $R^{*}$ ). The conclusion may then be drawn using the projection operation, valuating the existential quantifier by the supremum and the conjunction by the operation $\sqcap \in\{\wedge, *\}$. We obtain the definition of the composition of a fuzzy relation and a fuzzy set as

$$
\mu_{A^{*}}(u)=\bigvee_{v \in V}\left\{\mu_{R^{*}}(v, u) \sqcap \mu_{B^{*}}(v)\right\}, \forall u \in U
$$


In order to avoid the use of rule-misfiring, we modify the inference scheme in such a way that significant change will make the conclusion less specific. This is done by choosing an expansion type of inference scheme. Here, the "UNKNOWN" case, i.e., the fuzzy set $A^{*}=U$, is to be taken as the limit of non-specificity. Explicitly, when the similarity value becomes low, i.e., when not $B$ and $B^{*}$ differ significantly, the inference should be $A^{*}=U$. As for $B^{*}=$ not $B$, we expect that

$A^{*}=\operatorname{not} A$ and for all other $B^{*}$, the relation $A^{*} \supset \operatorname{not} A$ holds. This in turn implies that, nothing better than what the rule says, should be allowed as a valid conclusion. In view of the above observation we propose a scheme for computation of $A^{*}$ in the following algorithm.

\section{ALGORITHM-SIAR:}

Step 1. Translate given premise $p^{\prime}$ and compute $R(\operatorname{not} B, \operatorname{not} A)$ using some suitable translating rule (possibly, a t-norm).

Step 2. Compute similarity measure $S\left(\operatorname{not} B, B^{*}\right)$ using some suitable definition .

Step 3. Modify $R(\operatorname{not} B, \operatorname{not} A)$ with $S\left(\operatorname{not} B, B^{*}\right)$ to obtain the modified conditional relation $R\left(\right.$ not $B$, not $\left.A \mid B^{*}\right)$ using some scheme $C$.

Step 4. Use sup-projection operation on

$$
\begin{aligned}
R\left(\operatorname{not} B, \operatorname{not} A \mid B^{*}\right) & \text { to obtain } A^{*} \text { as } \\
\mu_{A^{*}}(u) & =\sup _{v} \mu_{R\left(\operatorname{not} B, \operatorname{not} A \mid B^{*}\right)}(v, u) .
\end{aligned}
$$

In $[17,18]$, authors have proposed two schemes for computation of the modified conditional relation $R\left(\operatorname{not} B, \operatorname{not} A \mid B^{*}\right)$ as given in Step 3, the general form of which is given by:

Scheme C:

$$
\mu_{R\left(\operatorname{not} B, \operatorname{not} A \mid B^{*}\right)}(v, u)=\left(s \rightarrow \mu_{R(\operatorname{not} B, \operatorname{not} A)}(v, u)\right),
$$

where $\rightarrow$ is any implication function. As previously done in [13], We have

$$
\mu_{A^{*}}(u)=\left(s \rightarrow \sup _{v \in V} \mu_{R(\operatorname{not} B, \operatorname{not} A)}(v, u)\right)
$$

and when the conditional relation is interpreted as a t-norm we get

$$
\mu_{A^{*}}(u)=\left(s \rightarrow \mu_{\text {not } A}(u)\right) .
$$

Otherwise, $A^{*}$ by SIAR could be anything. From (2) and (3) it is found that when $S\left(\operatorname{not} B, B^{*}\right)=0$ we have $A^{*}=U$. In other words, it is impossible to conclude anything when $\left\{\right.$ not $\left.B, B^{*}\right\}$ are completely dissimilar, i.e., $\left\{B, B^{*}\right\}$ are completely similar. When $S\left(\operatorname{not} B, B^{*}\right)$ is close to unity, $R\left(\operatorname{not} B, \operatorname{not} A \mid B^{*}\right)$ is close to $R(\operatorname{not} B, \operatorname{not} A)$. Hence, the inferred fuzzy set $A^{*}$ will be close to not $A$, i.e., $S\left(\operatorname{not} A, A^{*}\right)$ is close to unity. Scheme $C$ also ensures that a small change in the input produces a small change in the output and hence, in this sense the above mechanism of inference is reasonable. Let us consider the model as in Table $\mathbf{2}$ and a theorem is established as follows:

Theorem 1: For all not $B$ and $B^{*}$, not $A \subseteq A^{*}$.

We have investigated another method to deal with fuzzy implication operators in inverse approximate reasoning.

\subsection{Method Using Cylindrical Extension and Projection-INAR}

We now consider the scheme given in Table 2 and investigate the scheme for generalised modus tollens (GMT). We describe the method simply by an algorithm.

\section{ALGORITHM-INAR:}

Step 1. Translate the rule into a fuzzy relation or implication operator $R$.

Step 2. Take the cylindrical extension of fuzzy subset $B^{*}$ in $V$ on $U \times V$ and let it be $R^{\prime}$.

Step 3. Construct $R^{*}=R \cap R^{\prime}$, where $\cap$ is defined by any fuzzy conjunction operator.

Step 4. Obtain $A^{*}=\operatorname{proj} R^{*}$ on $U$.

Symbolically, we get,

$$
\begin{gathered}
\mu_{A^{*}}(u)=\operatorname{proj}_{v \in V} R^{*}(u, v) \\
=\sup _{v \in V} T\left(\mu_{R^{\prime}}(u, v), \mu_{R}(u, v)\right),
\end{gathered}
$$

$T$ is a t-norm

$$
=\sup _{v \in V} T\left(\mu_{B^{*}}(v), \mu_{R}(u, v)\right),
$$

by definition of cylindrical extension, which establishes the CRI in the form of GMT. We have to select an appropriate fuzzy implication for the fuzzy relation in Step 1 so as to model GMT. Also the standard negation of the resulted fuzzy set obtained by GMT also gives the given observation by applying GMP. Hence, mathematical formulation of the above algorithm is:

$$
\mu_{A^{*}}(u)=\sup _{v \in V} T\left(\mu_{B^{*}}(v), \mu_{R}(u, v)\right) .
$$

We now deduce some theorems from which we can establish the reasonableness of the method in which negation operator is taken as standard strong negation.

Theorem 2: Let $B^{*}$ be normal and $B^{*}=\operatorname{not} B$. Then not $A \subseteq A^{*}$, whenever the following implications satisfy the Equation (4) for any t-norm $T$ :

1) Reichenbach S-implication; 2) Kleene-Dienes $S$-implication and 3) Lukasiewicz $R$ and $S$-implication.

Theorem 3: Let $B^{*}$ be normal and $B^{*}=$ not $B$. Then $A^{*}=$ UNKNOWN, whenever both the relation and conjunction in the Equation (4) are defined by any t-norm $T$.

Theorem 4: Let $B^{*}$ be normal and $B^{*}=\operatorname{not} B$. Then not $A \supseteq A^{*}$, whenever the Rescher-Gaines R-implication $I$ satisfies the Equation (4) for any t-norm $T$. 
Theorem 5: Let $B^{*}$ be normal and $B^{*}=\operatorname{not} B$. Then not $A \subset A^{*}$, if $\mu_{A}(u) \geq 0.5$ and not $A \supseteq A^{*}$, if $\mu_{A}(u) \leq 0.5$, whenever Gödel R-implication and Gougen R-implication satisfy the Equation (4) for any t-norm $\mathrm{T}$.

We observe in the above theorems that if $B^{*}=\operatorname{not} B$ then either $A^{*}$ will be $\operatorname{not} A$ or close to not $A$, that is, we may use GMT in the case of inverse approximate reasoning to get an output in the antecedent part of the given rule and the negation of this output may give $B^{*}$ by applying GMP. So, we first consider the similarity between $B$ and $B^{*}$. If the similarity measure between these two is very very low, i.e., $S\left(B, B^{*}\right) \approx 0$ then we expect similarity between $A$ and $A^{*}$ to be very low, i.e., $S\left(A, A^{*}\right) \approx 0$. If the similarity measure between these two is very very high, i.e., $S\left(B, B^{*}\right) \approx 1$ then we cannot make any specific conclusion about the similarity between $A$ and $A^{*}$. Therefore, the method of inverse approximate reasoning demands dissimilarity between the specific observation and the consequent of the given rule. So we can apply the method in fuzzy resolution.

\section{Fuzzy Resolution Based on Inverse Approximate Reasoning}

Lately researchers discuss and explore the validity of many classical logic tautologies in fuzzy logic, especially those that involve fuzzy implications. We attempt to exploit such a classical logic equivalence to deal with fuzzy resolution in the framework of inverse approximate reasoning methodology. In classical logic

$$
a \rightarrow b \equiv \neg a \vee b, \forall a, b \in\{0,1\} .
$$

when extending this classical logic equivalence to fuzzy logic, we interpret the disjunction and negation as a fuzzy union (t-conorm) and a fuzzy complement, respectively. Fuzzy implication thus obtained is usually referred to in the literature as $S$-implication.

We now consider the classical logic tautology which is obtained from (5).

$$
a \vee b \equiv \neg a \rightarrow b, \forall a, b \in\{0,1\} .
$$

Therefore, we can extend the classical equivalence (6) into fuzzy logic where fuzzy union is transformed to fuzzy implication.

In fuzzy resolution we deal with the rule of the type “ $X$ is $A$ or $Y$ is $B$ ". Like classical logic, we may transform the rule into "If $X$ is not $A$ then $Y$ is $B$ " into fuzzy logic. Then the rule is executed in the method of Inverse Approximate Reasoning described in [13] to obtain the disjunct. The equivalent scheme of Table 1 that conforms fuzzy resolution is given in the Table 5.

We have demonstrated earlier in [13] that-if the give data is sufficiently dissimilar to the consequent part of a
Table 5. Equivalent scheme conforms fuzzy resolution.

\begin{tabular}{cc}
\hline Rule: If $X$ is not $A$ then & $Y$ is $B$ \\
\hline Fact: & $X$ is $B^{*}$ \\
Conclusion: $X$ is $A^{*}$ & \\
\hline
\end{tabular}

given rule then one may conclude that the resulting fuzzy set is sufficiently dissimilar to the antecedent part of the rule. Applying this method in the scheme given in Table 5, we get the required resolvent which establishes the fuzzy resolution principle. So the algorithm is as follows.

\section{ALGORITHM-FRIAR:}

Step 1. Translate the rule into fuzzy implication as

$$
\mu_{R}(u, v)=I\left(\mu_{\text {not } A}(u), \mu_{B}(v)\right)
$$

where $I$ is an implication operator.

Step 2. Take cylindrical extension of $B^{*}$ in $V$ on $U \times V$, say $R^{\prime}$, defined by

$$
R^{\prime}=\sum_{U \times V} \mu_{B}(v) /(u, v) .
$$

Step 3. Construct

$$
R^{*}=R \cap R^{\prime},
$$

where $\cap$ denotes any fuzzy conjunction operator.

Step 4. Obtain $A^{*}=\operatorname{proj} R^{*}$ on $U$ defined by

$$
\operatorname{proj} R^{*} \text { on } U=\sum_{u} \sup _{v} \mu_{R}(u, v) / v \text {. }
$$

Mathematically, we get

$$
\begin{aligned}
\mu_{A^{*}}(u) & =\operatorname{Proj}_{v \in V} R^{*}(u, v) \\
& =\sup _{v \in V} T\left(\mu_{R}(u, v), \mu_{R^{\prime}}(u, v)\right) \\
& =\sup _{v \in V} T\left(\mu_{R}(u, v), \mu_{B^{*}}(v)\right),
\end{aligned}
$$

where $T$ is a t-norm used to describe fuzzy conjunction operator.

It is expected that, for the observation " $Y$ is not $B$ " and the given premise " $X$ is $A$ or $Y$ is $B$ " we can conclude " $X$ is $A$ " by fuzzy resolution. However, for the the observation " $Y$ is $B$ " no conclusion can be drawn. We establish the above criteria by the following theorems.

Theorem 6: Let $B^{*}=\operatorname{not} B$ be normal and $R$ be interpreted by any S-implication satisfying Equation (7). Then $A^{*} \supseteq A$ for any t-norm $T$.

Proof: We prove the theorem only for Reichenbach S-implication and $T=\min$. Proofs for other implications are same as it.

$$
\begin{aligned}
& \mu_{A^{*}}(u) \\
= & \sup _{v \in V} T\left(\mu_{\text {not } B}(v), \mu_{A}(u)+\mu_{B}(v)-\mu_{A}(u) \cdot \mu_{B}(v)\right) \\
\geq & \sup _{v \in V} T\left(\mu_{\text {not } B}(v), \mu_{A}(u)-\mu_{A}(u) \cdot \mu_{B}(v)\right),
\end{aligned}
$$




$$
\begin{gathered}
\text { since } \mu_{B}(v) \geq 0 \text { and } T(a, c) \geq T(a, d) \\
\text { for } c \geq d, \forall a, c, d \in[0,1] \\
=T\left(\sup _{v \in V} \mu_{\text {not } B}(v), \mu_{A}(u) \cdot \sup _{v \in V} \mu_{\text {not } B}(v)\right)=\mu_{A}(u), \\
\text { since not } B \text { is normal and } \mu_{\text {not } B}(v)=1-\mu_{B}(v) .
\end{gathered}
$$

Corollary: Let $B^{*}=$ not $B$ be normal and $R$ be interpreted by any $\mathrm{S}$-implication satisfying Equation (7). Then $A^{*}=A$ for Lukasiewicz t-norm $T$.

\section{Proof:}

$$
\begin{aligned}
\mu_{A^{*}}(u)= & \sup _{v \in V} \sup \left\{0,1-\mu_{B}(v)+\mu_{A}(u)\right. \\
& \left.+\mu_{B}(v)-\mu_{A}(u) \cdot \mu_{B}(v)\right\} \\
= & \sup _{v \in V} \sup \left\{0, \mu_{A}(u) \cdot\left(1-\mu_{B}(v)\right)\right\} \\
= & \sup _{v \in V} \sup \left\{0, \mu_{A}(u) \cdot \mu_{\text {notB }}(v)\right\} \\
= & \mu_{A}(u)
\end{aligned}
$$

Example 2: Consider the premises

$$
\begin{aligned}
& p: X \text { is LARGE or } Y \text { is SMALL; } \\
& q: \quad Y \text { is not SMALL }
\end{aligned}
$$

in which $X$ and $Y$ are defined over the universes $U=\left\{u_{1}, \cdots, u_{4}\right\}$ and $V=\left\{v_{1}, \cdots, v_{4}\right\}$ respectively and fuzzy sets labelled by LARGE, SMALL and not SMALL are defined by

$$
\begin{aligned}
& A \square \mathrm{LARGE}=0 / u_{1}+0.45 / u_{2}+0.95 / u_{3}+1 / u_{4} \\
& B \square \mathrm{SMALL}=1 / v_{1}+0.65 / v_{2}+0.15 / v_{3}+0 / v_{4} \\
& B^{*} \square \operatorname{not} \mathrm{SMALL}=0 / v_{1}+0.35 / v_{2}+0.85 / v_{3}+1 / v_{4} .
\end{aligned}
$$

The similarity between fuzzy sets $B$ and $B^{*}$ is 0.0 , i.e., fuzzy set $B^{*}$ in observation is dissimilar to fuzzy set $B$ in the disjunctive form of rule.

Again, by INAR, we study the shape of the resolvent $A^{*}$ for data given in above with different $S$-implications and different t-norms, which is described in the Tables 6-8.

The result shows that the dissimilarity between $B^{*}$ and $B$ assures the similarity between $A^{*}$ and $A$ when the reasoning mechanism is handled using inverse approximate reasoning.

$\therefore$ "given a disjunction and the negation of one of the disjuncts, the other may be inferred" is established in fuzzy logic.

Example 3: Now, we consider the scheme and data of Example 2 except $B^{*}$. Consider

$B^{*}=0.0 / v_{1}+0.1225 / v_{2}+0.7225 / v_{3}+1.0 / v_{4}$ in Equation (4). We shall observe the results for the given premise " $p$ "and data in Example 2.

In this case, $S\left(B, B^{*}\right)=0.1304$, i.e., fuzzy sets $B$ and $B^{*}$ are dissimilar.
Table 6. $A^{*}$ for Reichenbach $S$-implication.

\begin{tabular}{ccccccc}
\hline$T / \mu_{A^{*}}$ & $u_{1}$ & $u_{2}$ & $u_{3}$ & $u_{4}$ & $A^{*}$ & $S\left(A, A^{*}\right)$ \\
\hline$M$ & 0.35 & 0.53 & 0.95 & 1.0 & $\supset A$ & 0.820 \\
$P$ & 0.23 & 0.45 & 0.95 & 1.0 & $\supset A$ & 0.886 \\
$B$ & 0.0 & 0.45 & 0.95 & 1.0 & $A$ & 1.0 \\
\hline
\end{tabular}

Table 7. $A^{*}$ for Kleene-Dienes $S$-implication.

\begin{tabular}{ccccccc}
\hline$T / \mu_{A^{*}}$ & $u_{1}$ & $u_{2}$ & $u_{3}$ & $u_{4}$ & $A^{*}$ & $S\left(A, A^{*}\right)$ \\
\hline$M$ & 0.35 & 0.45 & 0.95 & 1.0 & $\supset A$ & 0.825 \\
$P$ & 0.23 & 0.45 & 0.95 & 1.0 & $\supset A$ & 0.886 \\
$B$ & 0.0 & 0.45 & 0.95 & 1.0 & $A$ & 1.0 \\
\hline
\end{tabular}

Table 8. $A^{*}$ for Lukasiewicz $S$-implication.

\begin{tabular}{ccccccc}
\hline$T / \mu_{A^{*}}$ & $u_{1}$ & $u_{2}$ & $u_{3}$ & $u_{4}$ & $A^{*}$ & $S\left(A, A^{*}\right)$ \\
\hline$M$ & 0.35 & 0.60 & 0.95 & 1.0 & $\supset A$ & 0.809 \\
$P$ & 0.23 & 0.51 & 0.95 & 1.0 & $\supset A$ & 0.882 \\
$B$ & 0.0 & 0.45 & 0.95 & 1.0 & $A$ & 1.0 \\
\hline
\end{tabular}

Let us execute the reasoning mechanism by INAR. The results are shown in Tables 9-11 respectively for different implications and t-norms.

That is, if $B^{*}$ is not exactly match with not $B$ but these are dissimilar, the fuzzy resolvent can be obtained through inverse approximate reasoning method. The technique is very new one.

Theorem 7: Let $B^{*}=B$ be normal and $R$ be interpreted by any implication satisfying Equation (7). Then $A^{*}=\mathrm{UNKNOWN}$ for any t-norm $T$.

\section{Proof:}

$$
\begin{aligned}
& \mu_{A^{*}}(u) \\
& =\sup _{v \in V} T\left(\mu_{B}(v), \mu_{A}(u)+\mu_{B}(v)-\mu_{A}(u) \cdot \mu_{B}(v)\right) \\
& =\sup _{v \in V} \min \left(\mu_{B}(v), \mu_{A}(u)+\mu_{B}(v)-\mu_{A}(u) \cdot \mu_{B}(v)\right) \\
& =\sup _{v \in V} \mu_{B}(v)=1, \\
& \text { since } \mu_{A}(u) \cdot\left(1-\mu_{B}(v)\right) \geq 0 .
\end{aligned}
$$

Hence, $A^{*}=\mathrm{UNKNOWN}$.

We prove the theorem for Reichenbach S-implication and $T=\min$ only, but the above theorem can be proved for any other implications and any other t-norms in the similar way.

Example 4: In Example 2, if we take $B^{*}=B \square \mathrm{SMALL}=1.0 / v_{1}+0.65 / v_{2}+0.15 / v_{3}+0.0 / v_{4} \quad$ in Equation (4) then either by SIAR or by INAR we get $A^{*}=1.0 / u_{1}+1.0 / u_{2}+1.0 / u_{3}+1.0 / u_{4}=\mathrm{UNKNOWN}$, for 
Table 9. Another $A^{*}$ for Reichenbach $S$-implication.

\begin{tabular}{ccccccc}
\hline$T / \mu_{A^{*}}$ & $u_{1}$ & $u_{2}$ & $u_{3}$ & $u_{4}$ & $A^{*}$ & $S\left(A, A^{*}\right)$ \\
\hline$M$ & 0.15 & 0.53 & 0.95 & 1.0 & $\supset A$ & 0.9144 \\
$P$ & 0.11 & 0.45 & 0.95 & 1.0 & $\supset A$ & 0.9458 \\
$B$ & 0.0 & 0.45 & 0.95 & 1.0 & $A$ & 1.0 \\
\hline
\end{tabular}

Table 10. Another $A^{*}$ for Kleene-Dienes $S$-implication.

\begin{tabular}{ccccccc}
\hline$T / \mu_{A^{*}}$ & $u_{1}$ & $u_{2}$ & $u_{3}$ & $u_{4}$ & $A^{*}$ & $S\left(A, A^{*}\right)$ \\
\hline$M$ & 0.15 & 0.45 & 0.95 & 1.0 & $\supset A$ & 0.9250 \\
$P$ & 0.11 & 0.45 & 0.95 & 1.0 & $\supset A$ & 0.9458 \\
$B$ & 0.0 & 0.45 & 0.95 & 1.0 & $A$ & 1.0 \\
\hline
\end{tabular}

Table 11. Another $A^{*}$ for Lukasiewicz $S$-implication.

\begin{tabular}{ccccccc}
\hline$T / \mu_{A^{*}}$ & $u_{1}$ & $u_{2}$ & $u_{3}$ & $u_{4}$ & $A^{*}$ & $S\left(A, A^{*}\right)$ \\
\hline$M$ & 0.15 & 0.60 & 0.95 & 1.0 & $\supset A$ & 0.8939 \\
$P$ & 0.11 & 0.45 & 0.95 & 1.0 & $\supset A$ & 0.9458 \\
$B$ & 0.0 & 0.45 & 0.95 & 1.0 & $A$ & 1.0 \\
\hline
\end{tabular}

all of the cases.

Theorem 8: Let $B^{*}=$ not $B$ be normal and $R$ be interpreted by Rescher-Gaines R-implication satisfying Equation (7). Then $A^{*} \subseteq A$ for any t-norm $T$.

Proof:

$$
\begin{aligned}
& \mu_{A^{*}}(u) \\
= & \sup _{b \in[0,1]} T\left(1-b, I\left(\mu_{\text {notA }}(u), b\right)\right) \\
= & \max \left\{\sup _{\mu_{\operatorname{not} A}(u) \leq b} T(1-b, 1), \sup _{\mu_{\operatorname{not} A}(u)>b} T(1-b, 0)\right\} \\
= & \max \left\{\sup _{\mu_{\operatorname{not} A}(u) \leq b}(1-b), 0\right\}=\sup _{\mu_{\operatorname{not} A}(u) \leq b}(1-b) \leq \mu_{A}(u) .
\end{aligned}
$$

Example 5: For the data given in Example 2, applying INAR for Recher-Gaines R-implication combined with any t-norm $T$, we get the fuzzy resolvent $A^{*}$ as

$A^{*}=0.0 / u_{1}+0.35 / u_{2}+0.85 / u_{3}+1.0 / u_{4}$ which is a subset of $A$ and $S\left(A, A^{*}\right) \approx 0.929$. It establishes Theorem 8 .

Observation: In Example 2, for another

$B^{*}=0.0 / v_{1}+0.1225 / v_{2}+0.7225 / v_{3}+1.0 / v_{4} \subset \operatorname{not} B$, we also get the fuzzy resolvent

$A^{*}=0.0 / u_{1}+0.12 / u_{2}+0.72 / u_{3}+1.0 / u_{4} \subset A$ when we apply INAR with Rescher-Gaines R-implication. However, considering other R-implications, except, Lukasiewicz R-implication we cannot get such a fuzzy resolvent $A^{*}$ which is a subset of $A$ for the same input data, although the fuzzy resolvent obtained is significant.
We are now going to aplpply another method SIAR [13] to obtain fuzzy resolvent for the scheme given in Table 1. Let us consider another classical logic equivalence

$$
a \vee b \equiv b \vee a \equiv \neg b \rightarrow a
$$

The classical logic equivalence (8) can be extended in fuzzy logic with implication and negation function. Then we transform the rule in Table 1 into its equivalent form " $p^{1}$ : If $Y$ is not $B$ then $X$ is $A$ " over the domain of $[0,1]^{V \times U}$. A fuzzy rule may be defined by means of a conjunction for defining a fuzzy Cartesian product rather than in terms of a multivalued logic implication $[13,22]$.

Therefore, the rule in $p^{1}$ is transformed into fuzzy relation $R$ as

$$
\mu_{R}(v, u)=T\left(1-\mu_{B}(v), \mu_{A}(u)\right),
$$

where $T$ is a t-norm describing a fuzzy conjunction.

Now we can apply our method SIAR described in [13]. The algorithm is as follows:

\section{ALGORITHM-FRSIAR:}

Step 1. Translate given premise $p^{1}$ and compute $R(\operatorname{not} B, A)$ by Equation (9).

Step 2. Compute similarity measure $S\left(\operatorname{not} B, B^{*}\right)$ using some suitable definition .

Step 3. Modify $R(\operatorname{not} B, A)$ with $S\left(\operatorname{not} B, B^{*}\right)$ to obtain the modified conditional relation $R\left(\operatorname{not} B, A \mid B^{*}\right)$ using Scheme $C$ in (3).

Step 4. Use sup-projection operation on $R\left(\operatorname{not} B, A \mid B^{*}\right)$ to obtain $A^{*}$ as

$$
\mu_{A^{*}}(u)=\sup _{v} \mu_{R\left(\operatorname{not} B, A \mid B^{*}\right)}(v, u) .
$$

We shall illustrate the method applied here by some suitable examples.

Example 6: Let us consider the data in Example 2. For completely dissimilar $B^{*}$ with $B$ and for different t-norms $T$, the shapes of fuzzy resolvent $A^{*}$ in $U$ are studied here, when we apply SIAR. The subsequent results are shown in Table 12. In each case, it turns out exacltly the fuzzy set $A$ which corresponds 'LARGE.

Example 7: Consider the data in Example 2 where $B^{*}$ is not completely dissimilar with $B$, but dissimilarity exceeds certain threshold. Then, applying SIAR we observe the shapes of $A^{*}$ and compare it with given $A$ for different t-norms, which is shown in Table 13.

Since $S\left(A, A^{*}\right)=0.9255$, i.e., $A^{*}$ is almost similar to $A$, it establishes fuzzy resolution in reasoning.

In the above methods, we apllied INAR or SIAR when the disjunctive knoledge can be transformed into fuzzy implication. However, it may not always be the case. Moreover, when the expert knowledge is in complex form of disjunction it is difficult to apply INAR or SIAR. So, we extend our method in such a way that can deal with complex premises. 
Table 12. Fuzzy resolvent $A^{*}$.

\begin{tabular}{ccccccc}
\hline$T / \mu_{A^{*}}$ & $u_{1}$ & $u_{2}$ & $u_{3}$ & $u_{4}$ & $A^{*}$ & $S\left(A, A^{*}\right)$ \\
\hline$M$ & 0.0 & 0.45 & 0.95 & 1.0 & $A$ & 1.0 \\
$P$ & 0.0 & 0.45 & 0.95 & 1.0 & $A$ & 1.0 \\
$B$ & 0.0 & 0.45 & 0.95 & 1.0 & $A$ & 1.0 \\
\hline
\end{tabular}

Table 13. $A^{*}$ for another $B^{*}$.

\begin{tabular}{ccccccc}
\hline$T / \mu_{A^{*}}$ & $u_{1}$ & $u_{2}$ & $u_{3}$ & $u_{4}$ & $A^{*}$ & $S\left(A, A^{*}\right)$ \\
\hline$M$ & 0.13 & 0.52 & 0.96 & 1.0 & $\supset A$ & 0.92 \\
$P$ & 0.13 & 0.52 & 0.96 & 1.0 & $\supset A$ & 0.92 \\
$B$ & 0.13 & 0.52 & 0.96 & 1.0 & $\supset A$ & 0.92 \\
\hline
\end{tabular}

\section{Fuzzy Resolution with Complex Clauses}

In this section, we shall extend the scheme given in Table 1. Let $X, Y$ and $Z$ be three linguistic variables that take values from the domain $U, V$ and $W$ respectively. We consider the derivation of an inexact conclusion " $r$ " from two typical knowledge (premises) " $p$ " and " $q$ " acording to the scheme given in Table 14, where $A$ 's, $B$ 's and $C$ 's are approximations of possibly inexact concepts by fuzzy sets over $U, V$ and $W$ respectively.

In 1993, Raha and Ray [12] applied Zadeh's [23] concept of approximate reasoning with the application of possibility theory to model a deductive process "Generalised Disjunctive Syllogism". They used projection principle and conjunction principle to deduce fuzzy resolvent. However, the method could not reduce the shortfall of Zadeh's Compositional rule inference. We have found the shortfalls in [12] as follows:

1) Let fuzzy resolvent be $R$ for $B^{\prime}=\operatorname{not} B$ in the scheme given in Table 14, by the method of Raha and Ray [12]. The fuzzy resolvent also be same $R$ for taking $B^{\prime}$ as either $B^{\prime} \subset \operatorname{not} B$ or $B^{\prime} \supset \operatorname{not} B$.

2) If we interchange $B$ and $B^{\prime}$ the same fuzzy resolvent $R$ will be produced.

Therefore, firing a rule on the basis of the observation could be harmful. So, it is necessary to modify the relation generated by the two given premises, with the similarity measure of two fuzzy sets involved in the disjunctive form of rules so that the shortfall can be removed. If a pair of fuzzy sets involved having dissimilarity greater than certain pre-defined threshold value, we get our expected fuzzy resolvent using some deductive reasoning. Hence, we investigate another method which is described in the following algorithm.

\section{ALGORITHM-FRCEP:}

Step 1. Translate the premise $p$ into fuzzy relation

$$
R_{1} \subseteq \mathcal{F}(U \times V)
$$

Table 14. Generalised fuzzy resolution—extended form.

\begin{tabular}{ccc}
$p:$ & $X$ is $A$ or & $Y$ is $B$ \\
$q:$ & $Y$ is $B^{\prime}$ or & $Z$ is $C$ \\
$r:$ & $X$ is $A^{\prime}$ or & $Z$ is $C^{\prime}$ \\
\hline
\end{tabular}

as

$$
\mu_{R_{1}}(u, v)=\min \left(\mu_{A}(u), 1-\mu_{B}(v)\right) ;
$$

Step 2. Translate the premise $q$ into fuzzy relation

$$
R_{2} \subseteq \mathcal{F}(V \times W)
$$

as

$$
\mu_{R_{2}}(v, w)=\min \left(\mu_{B^{\prime}}(v), \mu_{C}(w)\right) ;
$$

Step 3. Take cylindrical extension of $R_{1}$ in $U \times V$ on $U \times V \times W$, say $R_{1}^{\prime}$, defined by

$$
R_{1}^{\prime}=\sum_{U \times V \times W} \mu_{R_{1}}(u, v) /(u, v, w) \text {; }
$$

Step 4. Take cylindrical extension of $R_{2}$ in $V \times W$ on $U \times V \times W$, say $R_{2}^{\prime}$, defined by

$$
R_{2}^{\prime}=\sum_{U \times V \times W} \mu_{R_{2}}(v, w) /(u, v, w) ;
$$

Step 5. Construct $R^{\prime}=R_{1}^{\prime} \cap R_{2}^{\prime}$, where $\cap$ denotes any fuzzy conjunction operator;

Step 6. Compute $S\left(\operatorname{not} B, B^{\prime}\right)$ and, say, $s$;

Step 7. Modify $R^{\prime}$ with $S$ by Scheme $C$ in (3) and, say, $R^{*}$;

Step 8. Obtain $R=p r o j R^{*}$ on $U \times W$ defined by

$$
\operatorname{projR}^{*} \text { on } U \times W=\sum_{U \times W} \sup _{v} \mu_{R^{*}}(u, v, w) /(u, w) \text {; }
$$

Step 9. Obtain $A^{\prime}$ and $C^{\prime}$ by projecting $R$ separately on $U$ and $W$ such that $A^{\prime}=\operatorname{proj}_{U} R=\sum_{U} \sup _{w} \mu_{R}(u, w) / u$ and

$$
C^{\prime}=\operatorname{proj}_{W} R=\sum_{W} \sup _{u} \mu_{R}(u, w) / w
$$

Symbolically, the fuzzy resolvent $R$ is obtained by, for $u \in U, v \in V$ and $w \in W$,

$$
\begin{aligned}
& \mu_{R}(u, w)=\sup _{v} \mu_{R^{*}}(u, v, w) \\
& =\sup _{v}\left\{s \rightarrow \mu_{R^{\prime}}(u, v, w)\right\}, \operatorname{from}(3) \\
& =s \rightarrow \sup _{v} \mu_{R^{\prime}}(u, v, w) \\
& =s \rightarrow \sup _{v} T\left(\mu_{R_{1}^{\prime}}(u, v, w), \mu_{R_{2}^{\prime}}(u, v, w)\right) \\
& =s \rightarrow \sup _{v} T\left(\mu_{R_{1}}(u, v), \mu_{R_{2}}(v, w)\right) \\
& =s \rightarrow \sup _{v} T\left(\min \left(\mu_{A}(u), 1-\mu_{B}(v)\right),\right. \\
& \left.\qquad \min \left(\mu_{B^{\prime}}(v), \mu_{C}(w)\right)\right) \\
& =s \rightarrow T\left(\min \left(\mu_{A}(u), 1-\inf _{v} \mu_{B}(v)\right),\right. \\
& \left.\quad \min \left(\sup _{v} \mu_{B^{\prime}}(v), \mu_{C}(w)\right)\right) \\
& =s \rightarrow T\left(\mu_{A}(u), \mu_{C}(w)\right),
\end{aligned}
$$




$$
\text { iff } 1-\inf _{v} \mu_{B}(v)=\sup _{v} \mu_{B^{\prime}}(v)=1 .
$$

This derivation can be achieved if there is a $v_{0} \in V$ such that $\mu_{B}\left(v_{0}\right)=0$ and $\mu_{B^{\prime}}\left(v_{0}\right)=1$ which is possible if the fuzzy sets $B$ and $B^{\prime}$ are dissimilar, i.e., not $B$ and $B$ are similar for any implication $\rightarrow$ in derivation. We observe two criteria here.

Criterion 1: Taking $x \rightarrow y=\min (1, y / x)$, we get

$$
\mu_{R}(u, w)=\min \left(1, T\left(\mu_{A}(u), \mu_{C}(w)\right) / s\right) ;
$$

Criterion 2: Taking $x \rightarrow y=1-x+x y$, we get

$$
\mu_{R}(u, w)=1-s+T\left(\mu_{A}(u), \mu_{C}(w)\right) \cdot s .
$$

From above two criteria we observe that when $s=S\left(\operatorname{not} B, B^{\prime}\right)=0$, i.e., when $B$ and $B^{\prime}$ are completely similar $R=U \times W=\mathrm{UNKNOWN}$. Therefore, fuzzy resolvent could be anything. However, if $s$ is close to unity, i.e., if $B$ and $B^{\prime}$ are almost dissimilar we have $R$ is close to

$$
\sum_{U \times W} T\left(\mu_{A}(u), \mu_{C}(w)\right) /(u, w)
$$

which, after re-translation, gives "If $X$ is $A$ or $Z$ is $C$ ". Again, we observe that a small change in $B^{\prime}$ produces a small change in fuzzy resolvent-which ensures our method is resonable one.

Let us now investigate another method to find a fuzzy resolvent from a complex set of clauses. It is observed that, to obtain a fuzzy resolvent from two clauses containing a pair of complementary literals defined over the same domain. If the dissimilarity measure of the complementary literals is unity then we get the resolvent by the disjunction of remaining literals and subsequent removal of the complementary literals. The keyword of the fuzzy resolvent is any one of the complementary literals, the degree of confidence of which is measured by the dissimilarity measure of the complementary literals. However, in the case of almost complementary literals for which dissimilarity value does not attain unity, but exceeds a certain pre-defined threshold, we modify the remaining literals with the measure of dissimilarity in such a way that dissimilarity measure of complementary literals tends to unity and we obtain the resolvent by taking the disjunction of modified literals removing the complementary literals. If the dissimilarity measure of a pair of literals is either zero or close to zero, we cannot obtain a fuzzy resolvent. In this way, we can proceed for a method to find out the derivation of the empty clause and establish the refutation method for the proof of a theorem. The degree of confidence of the empty clause is measured by the degree of confidence of keyword that generated the empty clause and, thus, a sort of completeness of fuzzy resolution principle is established.

Let us consider a scheme given in Table $\mathbf{1 5}$ where
Table 15. Generalised fuzzy resolution-another extension.

$$
\begin{gathered}
C_{1}: X_{1} \text { is } A_{1} \text { or } X_{2} \text { is } A_{2} \text { or } \cdots \text { or } X_{m} \text { is } A_{m} ; \\
C_{2}: Y_{1} \text { is } B_{1} \text { or } Y_{2} \text { is } B_{2} \text { or } \cdots \text { or } Y_{n} \text { is } B_{n} \text {; } \\
R\left(C_{1}, C_{2}\right): X_{1} \text { is } A_{1}^{\prime} \text { or } \cdots \text { or } X_{m} \text { is } A_{m}^{\prime} \\
\quad \text { or } Y_{1} \text { is } B_{1}^{\prime} \text { or } \cdots \text { or } Y_{n} \text { is } B_{n}^{\prime}
\end{gathered}
$$

variables $X_{i}(i=1,2, \cdots, m)$ and the respective fuzzy subsets $A_{i}(i=1,2, \cdots, m)$ are defined on universe $U_{i}(i=1,2, \cdots, m)$ repectively; variables $Y_{j}(j=1,2, \cdots, n)$ and the respective fuzzy subsets $B_{j}(j=1,2, \cdots, n)$ are defined on universe $V_{j}(j=1,2, \cdots, n)$ repectively. $\left(A_{k}, B_{l}\right)$ is almost complementary over the same universe $U_{k}\left(=V_{l}\right)$ with the degree of confidence of keyword $A_{k}$ is $c d\left(A_{k}\right)=1-S\left(A_{k}, B_{l}\right)$ and the corresponding $A_{i}, B_{j}$ are defined over $U_{i}, V_{j}$ respectively.

To show the method we set up an algorithm FRAE as follows:

\section{ALGORITHM-FRAE:}

Step 1. Check the domain of pair of literals $\left(A_{i}, B_{j}\right)$, $\forall i, j$ from clauses $C_{1}$ and $C_{2}$;

Step 2. If the pair remains in the same domain, say, $U_{k}\left(V_{l}\right)$ then measure the dissimilarity of $\left(A_{k}, B_{l}\right)$; Otherwise, there is no fuzzy resolvent;

Step 3. Dissimilarity of $\left(A_{k}, B_{l}\right)$, i.e., $D\left(A_{k}, B_{l}\right)$ is computed by $1-S\left(A_{k}, B_{l}\right)$;

Step 4. If $D\left(A_{k}, B_{l}\right)$ is very very high, i.e., $D\left(A_{k}, B_{l}\right)>\epsilon, \epsilon$ is pre-defined threshold then go to the next step and say, $A_{k}$ is keyword; Otherwise, there is no fuzzy resolvent;

Step 5. Modify $B_{i}$ either by $B_{j}^{\prime}=\min \left(1, B_{j} / D\left(A_{k}, B_{l}\right)\right)$ or by $B_{j}^{\prime}=1-\left(1-B_{j}\right) \cdot S\left(A_{k}, B_{l}\right)$;

Step 6. Fuzzy resolvent is

$$
R\left(C_{1}, C_{2}\right)=A_{1}^{\prime} \vee \cdots \vee A_{m}^{\prime} \vee B_{1}^{\prime} \vee \cdots \vee B_{n}^{\prime}
$$

and

$$
R\left(C_{1}, C_{2}\right)_{c d}=c d\left(A_{k}\right)
$$

which is measured as $c d\left(A_{k}\right)=D\left(A_{k}, B_{l}\right)$;

Step 7. Repeat the process until empty clause, with the confidence $c d \neq 0$, is derived for more than two clauses.

Hence, we prove the (un)satisfiability of a theorem by the deduction of empty clause from a set of fuzzy clauses.

\section{Artificial Examples}

We consider examples to illustrate the models presented in this paper. Let us consider variables that range over finite sets or can be approximated by variables ranging over such sets.

Example 8: Consider the premises 


\section{$p: X$ is LARGE or $Y$ is SMALL; $q: Y$ is not SMALL or $Z$ is LARGE;}

in which $X, Y$ and $Z$ are defined over the respective universes $U=\left\{u_{1}, \cdots, u_{4}\right\}, \quad V=\left\{v_{1}, \cdots, v_{4}\right\}$ and $W=\left\{w_{1}, w_{2}, w_{3}, w_{4}\right\}$ and fuzzy sets labelled by

LARGE, SMALL and not SMALL are defined by

$$
\begin{aligned}
& A \square \operatorname{LARGE}=0 / u_{1}+0.45 / u_{2}+0.95 / u_{3}+1 / u_{4} \\
& B \square \operatorname{SMALL}=1 / v_{1}+0.65 / v_{2}+0.15 / v_{3}+0 / v_{4} \\
& B^{\prime} \square \operatorname{not} \text { SMALL }=0 / v_{1}+0.35 / v_{2}+0.85 / v_{3}+1 / v_{4} \\
& C \square \operatorname{LARGE}=0 / w_{1}+0.20 / w_{2}+0.75 / w_{3}+1 / w_{4} .
\end{aligned}
$$

The similarity between fuzzy sets not $B$ and $B^{\prime}$ is 1.0 , i.e., $S\left(\right.$ not $\left.B, B^{\prime}\right)=1.0$. Therefore, in two clauses, fuzzy sets $B^{\prime}$ in observation is dissimilar to fuzzy set $B$. Hence, we can resolve upon dissimilar pair $\left\{B, B^{\prime}\right\}$. We apply ALGORITHM-FRCEP to get the resolvent.

$$
\begin{aligned}
R_{1} & =\sum_{U \times V} \min \left(\mu_{A}(u), 1-\mu_{B}(v)\right) /(u, v) \\
& =\left(\begin{array}{llll}
0.00 & 0.00 & 0.00 & 0.00 \\
0.00 & 0.35 & 0.45 & 0.45 \\
0.00 & 0.35 & 0.85 & 0.95 \\
0.00 & 0.35 & 0.85 & 1.00
\end{array}\right), \\
R_{2} & =\sum_{V \times W} \min \left(\mu_{B^{\prime}}(v), \mu_{C}(w)\right) /(v, w) \\
& =\left(\begin{array}{llll}
0.00 & 0.00 & 0.00 & 0.00 \\
0.01 & 0.20 & 0.35 & 0.35 \\
0.01 & 0.20 & 0.75 & 0.85 \\
0.01 & 0.20 & 0.75 & 1.00
\end{array}\right) .
\end{aligned}
$$

We construct $R^{\prime}=R_{1}^{\prime} \cap R_{2}^{\prime}$, where $\cap$ denotes any fuzzy conjunction operator and

$$
\begin{aligned}
& R_{1}^{\prime}=\sum_{U \times V \times W} \mu_{R_{1}}(u, v) /(u, v, w), \\
& R_{2}^{\prime}=\sum_{U \times V \times W} \mu_{R_{2}}(v, w) /(u, v, w) .
\end{aligned}
$$

We modify $R^{\prime}$ with $s=S\left(\right.$ not $\left.B, B^{\prime}\right)$ as we get $\mu_{R^{*}}(u, v, w)=1-\left(1-\mu_{R^{\prime}}\right) \cdot s \quad$ (using Scheme $C_{2}$ and $\cap \equiv M \quad$ in Table 3$)$ and we get

$$
\begin{aligned}
R & =\operatorname{proj}^{*} \text { on } U \times W \\
& =\left(\begin{array}{llll}
0.00 & 0.00 & 0.00 & 0.00 \\
0.01 & 0.20 & 0.45 & 0.45 \\
0.01 & 0.20 & 0.75 & 0.95 \\
0.01 & 0.20 & 0.75 & 1.00
\end{array}\right) .
\end{aligned}
$$

We can also obtain $A^{\prime}$ and $C^{\prime}$ by

$$
\begin{gathered}
A^{\prime}=\operatorname{proj}_{U} R \\
=0.0 / u_{1}+0.45 / u_{2}+0.95 / u_{3}+1.0 / u_{4}, \\
C^{\prime}=\operatorname{proj}_{W} R=0.0 / w_{1}+0.20 / w_{2}+0.75 / w_{3}+1.0 / w_{4},
\end{gathered}
$$

which are same as $A$ and $B$ respectively, since the dissimilarity between $B$ and $B^{\prime}$ is zero.

Again, it is observed that same $R, A^{\prime}$ and $C^{\prime}$ are obtained for different fuzzy conjunctions (t-norms, here) described in Table 3. However, for different $B^{\prime}$ in premise $q$, it yields different results.

Let us consider

$B^{\prime}=0.0 / v_{1}+0.1225 / v_{2}+0.7225 / v_{3}+1.0 / v_{4} \subset B$.

Similarity between not $B$ and $B^{\prime}$ is 0.869604 , i.e., dissimilarity between $B$ and $B^{\prime}$ is very low, being less than pre-defined threshold value 0.34(1-0.86). By applying ALGORITHM-FRCEP, we get the fuzzy resolvent as

$$
R=\left(\begin{array}{llll}
0.13 & 0.13 & 0.13 & 0.13 \\
0.14 & 0.30 & 0.52 & 0.52 \\
0.14 & 0.30 & 0.78 & 0.96 \\
0.14 & 0.30 & 0.78 & 1.00
\end{array}\right),
$$

for t-norms M, P and B in Table 3, and by projection we obtain

$$
\begin{aligned}
& A^{\prime}=0.13 / u_{1}+0.52 / u_{2}+0.96 / u_{3}+1.0 / u_{4}, \\
& C^{\prime}=0.14 / w_{1}+0.30 / w_{2}+0.78 / w_{3}+1.0 / w_{4} .
\end{aligned}
$$

Similarity between $A$ and $A^{\prime}$ is 0.925520 , and similarity between $C$ and $C^{\prime}$ is 0.915428 which is our expected result.

Now, if we consider another $B^{\prime}=0.0 / v_{1}+0.5916 / v_{2}+0.9219 / v_{3}+1.0 / v_{4} \supset B$, similarity of which with not $B$ is 0.873964 , i.e., dissimilarity of it with $B$ is less than $1-0.86$ then, we find the resolvent as

$$
R=\left(\begin{array}{llll}
0.13 & 0.13 & 0.13 & 0.13 \\
0.13 & 0.30 & 0.52 & 0.52 \\
0.13 & 0.30 & 0.78 & 0.96 \\
0.13 & 0.30 & 0.78 & 1.00
\end{array}\right),
$$

for different t-norms in Table 3 and

$$
\begin{aligned}
& A^{\prime}=0.13 / u_{1}+0.52 / u_{2}+0.96 / u_{3}+1.0 / u_{4}, \\
& C^{\prime}=0.14 / w_{1}+0.30 / w_{2}+0.78 / w_{3}+1.0 / w_{4}
\end{aligned}
$$

with $S\left(A, A^{\prime}\right)=0.928010$ and $S\left(C, C^{\prime}\right)=0.918256$.

Another example can be illustrated whenever number of premises is more than two.

Example 9: Let us consider the premises

$$
\begin{aligned}
& p: X \text { is LARGE or } Y \text { is SMALL or } Z \text { is LARGE; } \\
& q: X \text { is not LARGE; } \\
& r: Y \text { is not SMALL; }
\end{aligned}
$$

where $X, Y, Z$ and all the fuzzy sets are defined as earlier. Here, not LARGE is defined by

$$
A^{\prime} \square \text { not LARGE }=1.0 / u_{1}+0.55 / u_{2}+0.05 / u_{3}+0.0 / u_{4} .
$$

Selection of keywords: Since, $A \square$ LARGE in pre- 
mise $p$ and $A^{\prime} \square$ not LARGE in premise $q$ are fuzzy subsets in the same universe $U$ and similarity between not $A$ and $A^{\prime}$, i.e., $S\left(\operatorname{not} A, A^{\prime}\right)=1$, we resolve upon the keyword $A$, taking the premises $p$ and $q$ together as $A$ and $A^{\prime}$ completely dissimilar. Again, $B \square$ SMALL in premise $p$ and $B^{\prime} \square$ not SMALL in premise $r$ are fuzzy subsets over the same universe $V$ and $S\left(\right.$ not $\left.B, B^{\prime}\right)=1$. Therefore, after resolving with $p$ and $q$ we resolve upon the keyword $B$ with taking $r$ and $\operatorname{res}(p, q)$ together. Next, we execute the following steps with the given data, using ALGORITHM- FRCEP.

\section{Execution:}

Step 1: Compute the fuzzy relation $R_{1}=A \vee B \vee C$ by

$$
\mu_{R_{1}}(u, v, w)=\min \left(1-\mu_{A}(u), 1-\mu_{B}(v), \mu_{C}(w)\right) ;
$$

Step 2: Extend $A^{\prime}$ in $U$ cylindrically on $U \times V \times W$ as

$$
R_{2}=\sum_{U \times V \times W} \mu_{A^{\prime}}(u) /(u, v, w) ;
$$

Step 3: Compute $R^{\prime}=R_{1} \cap R_{2}$ by

$$
\mu_{R^{\prime}}(u, v, w)=T\left(\mu_{R_{1}}(u, v, w), \mu_{R_{2}}(u, v, w)\right),
$$

taking fuzzy conjunction $\cap$ as t-norm $T$;

Step 4: Modify $R^{\prime}$ with $S\left(1-\mu_{A}(u), \mu_{A^{\prime}}(u)\right)=s_{1}$ to get $R^{*}$ as

$$
\mu_{R^{*}}(u, v, w)=1-\left(1-s_{1}\right) \cdot \mu_{R^{\prime}}(u, v, w) ;
$$

Step 5: Project $R^{*}$ on $V \times W$ such that

$$
\begin{aligned}
R & =\sum_{V \times W} \sup _{u} \mu_{R^{*}}(u, v, w) \\
& =\left(\begin{array}{llll}
0.00 & 0.00 & 0.00 & 0.00 \\
0.00 & 0.20 & 0.45 & 0.45 \\
0.00 & 0.20 & 0.75 & 0.85 \\
0.00 & 0.20 & 0.75 & 1.00
\end{array}\right) ;
\end{aligned}
$$

Step 6: Extend $B^{\prime}$ in $V$ cylindrically on $V \times W$ as

$$
R_{3}=\sum_{V \times W} \mu_{B^{\prime}} /(v, w) ;
$$

Step 7: Compute $R^{\prime \prime}=R \cap R_{3}$ by

$$
\mu_{R^{\prime \prime}}(v, w)=T\left(\mu_{R}(v, w), \mu_{R_{3}}(v, w)\right),
$$

taking fuzzy conjunction $\cap$ as t-norm $T$;

Step 8: Modify $R^{\prime \prime}$ with $S\left(1-\mu_{B}(v), \mu_{B^{\prime}}(v)\right)=s_{2}$ to get $R^{* *}$ as

$$
\mu_{R^{* *}}(v, w)=1-\left(1-s_{2}\right) \cdot \mu_{R^{n}}(v, w) ;
$$

Step 9: Project $R^{* *}$ on $W$ such that

$$
\begin{aligned}
C^{\prime} & =\sum_{W} \sup _{v} \mu_{R^{* *}}(v, w) \\
& =0.0 / w_{1}+0.20 / w_{2}+0.75 / w_{3}+1.0 / w_{4},
\end{aligned}
$$

which is completely similar to $C$, i.e., $S\left(C^{\prime}, C\right)=1$.

Even if $A^{\prime}$ and $B^{\prime}$ in the respective premises $p$ and $q$, are not completely dissimilar to $A$ and $B$ respectively, but the dissimilarity measures attain values greater than certain pre-defined threshold then we can get a fuzzy resolvent $C^{\prime}$ which is almost similar to $C$, using FRCEP.

$$
\begin{gathered}
\text { Suppose, } A^{\prime}=1.0 / u_{1}+0.3025 / u_{2}+0.0025 / u_{3}+0.0 / u_{4} ; \\
B^{\prime}=0.0 / v_{1}+0.1225 / v_{2}+0.7225 / v_{3}+1.0 / v_{4} . \\
S\left(\operatorname{not} A, A^{\prime}\right)=0.873992 ; S\left(\operatorname{not} B, B^{\prime}\right)=0.869604
\end{gathered}
$$
are computed.

Then, it yields

$$
C^{\prime}=0.24 / w_{1}+0.39 / w_{2}+0.81 / w_{3}+1.0 / w_{4}
$$

with $S\left(C, C^{\prime}\right)=0.843441$.

Therefore, it is possible to get a fuzzy resolvent from a set of fuzzy clauses if there is a pair of dissimilar literals contained in the respective clauses.

\section{Conclusion}

This paper presented a resolution principle for fuzzy formulae based on similarity and approximate reasoning methodology. Similarity is inherent in approximate resoning and resolution deduction can be used as a rule of inference to generate new clause from a given set of clauses. The essential idea of resolution of two clauses is to search for a literal in a clausal formula that is almost complementary to a literal in the other form. The clause formed by the disjunction of the remaining literals and subsequent removal of the pair of almost complementary literals is a logical consequence. If we put the resolvents in the set of clauses its behaviour (satisfiability) never changes. It can be applied directly to any set $S$ of clausal formulae (not necessarily to ground clauses) to test the (un)satisfiability of $S$. To test the unsatisfiability it checks whether $S$ contains the empty clause (as a resolution deduction). This could be a powerful technique in constructing a proof of a theorem using refutation procedure. Examples cited in the paper attempted to demonstrate how resolution can be effectively used to construct a proof of a theorem. Inverse approximate reasoning may be applied to model different goal-directed search techniques. We apply inverse approximate reasoning method to avoid the inherent problem of GMP. Instead of testing complementary literals we use dissimilarity concept of fuzzy literals.

\section{Acknowledgements}

This research has been partially supported by the UGC SAP (DRS) Phase-II project under the Department of Mathematics, Visva-Bharati. 


\section{REFERENCES}

[1] J. A. Robinson, "A Machine Oriented Logic Based on the Resolution Principle," Journal of the ACM, Vol. 12, No. 1, 1965, pp. 23-41. doi:10.1145/321250.321253

[2] J. J. Kelly, "The Essence of Logic," Prentice-Hall, New Delhi, 1997.

[3] R. C. T. Lee and C. L. Chang, "Some Properties of Fuzzy Logic," Information and Control, Vol. 19, No. 1, 1971, pp. 417-431. doi:10.1016/S0019-9958(71)90684-X

[4] R. C. T. Lee, "Fuzzy Logic and the Resolution Principle," Journal of the ACM, Vol. 19, No. 1, 1972, pp. 109-119. doi:10.1145/321679.321688

[5] D. Dubois, J. Lang and H. Prade, "Fuzzy Sets in Approximate Reasoning, Part 2: Logical Approaches," Fuzzy Sets and Systems, Vol. 40, No. 1, 1991, pp. 203-244. doi:10.1016/0165-0114(91)90051-Q

[6] Z. Shen, L. Ding and M. Mukaidono, "Fuzzy Resolution Principle," Proceedings of the 18th International Symposium on Multivalued Logic, Palma de Mallorca, 24-26 May 1988, pp. 210-215. doi:10.1109/ISMVL.1988.5176

[7] M. Mukaidono, "Fuzzy Inference of Resolution Style," In: R. R. Yager, Ed., Fuzzy Set and Possibility Theory, Pergamon Press, New York, 1988, pp. 224-231.

[8] D. Dubois and H. Prade, "Necessity and the Resolution Principle," IEEE Transactions on Systems, Man and Cybernetics, Vol. 17, No. 3, pp. 474-478.

[9] C. S. Kim, D. S. Kim and J. Park, "A New Fuzzy Resolution Principle Based on the Antonym," Fuzzy Sets and Systems, Vol. 113, No. 2, 2000, pp. 299-307. doi:10.1016/S0165-0114(98)00063-3

[10] M. A. C. Viedma, R. M. Morales and I. N. Sanchez, "Fuzzy Temporal Constraint Logic: A Valid Resolution Principle," Fuzzy Sets and Systems, Vol. 117, No. 2, 2001, pp. 231-250. doi:10.1016/S0165-0114(99)00099-8

[11] F. A. Fontana and F. Formato, "A Similarity-Based Resolution Principle," International Journal of Intelligent Systems, Vol. 17, No. 9, 2002, pp. 853-872. doi:10.1002/int.10067

[12] S. Raha and K. S. Ray, "Approximate Reasoning Based on Generalised Disjunctive Syllogism," Fuzzy Sets and Systems, Vol. 61, No. 2, 1994, pp. 143-151.

[13] B. Mondal and S. Raha, "Similarity-Based Inverse Approximate Reasoning," IEEE Transaction on Fuzzy Systems, Vol. 19, No. 6, 2011, pp. 1058-1071.
doi:10.1109/TFUZZ.2011.2159981

[14] B. Lazzerini and F. Marcelloni, "Some Considerations on Input and Output Partitions to Produce Meaningful Conclusions in Fuzzy Inference," Fuzzy Sets and Systems, Vol. 113, No. 2, 2000, pp. 221-235. doi:10.1016/S0165-0114(98)00096-7

[15] B. Bouchon-Meunier, M. Rifqi and S. Bothorel, "Towards General Measures of Comparison of Objects," Fuzzy Sets and Systems, Vol. 84, No. 2, 1996, pp. 143-153. doi:10.1016/0165-0114(96)00067-X

[16] R. Zwick, E. Carlstein and D. V. Budescu, "Measures of Similarity among Fuzzy Concepts: A Comparative Analysis," International Journal of Approximate Reasoning, Vol. 1, No. 2, 1987, pp. 221-242. doi:10.1016/0888-613X(87)90015-6

[17] B. Mondal, D. Mazumdar and S. Raha, "Similarity in Approximate Reasoning," International Journal of Computational Cognition, Vol. 4, No. 3, 2006, pp. 46-56.

[18] S. Raha, N. R. Pal and K. S. Ray, "Similarity Based Approximate Reasoning: Methodology and Application," IEEE Transactions on Systems, Man and Cybernatics, Part A: Systems and Humans, Vol. 32, No. 4, 2002, pp. 541-547. doi:10.1109/TSMCA.2002.804787

[19] N. Mellouli and B. Bouchon-Meunier, "Fuzzy Approaches of Abductive Inference," Proceedings of the 8th International Workshop Non-Monotonic Reasoning, Breikenridge, 2000.

[20] N. Mellouli and B. Bouchon-Meunier, "Abductive Reasoning and Measure of Similitude in the Presence of Fuzzy Rules," Fuzzy Sets and Systems, Vol. 137, No. 1, 2003, pp. 177-188. doi:10.1016/S0165-0114(02)00439-6

[21] F. Klawonn and J. L. Castro, "Similarity in Fuzzy Reasoning," Mathware and Soft Computing, Vol. 3, 1995, pp. 197-228.

[22] L. Ughetto, D. Dubois and H. Prade, "Implicative and Conjunctive Fuzzy Rule-A Tool for Reasoning from Knowledge and Examples," Proceedings of the 16th National Conference on Artificial Intelligence and the 11th Innovative Applications of Artificial Intelligence Conference, American Association for Artificial Intelligence, Menlo Park, 1999, pp. 214-219.

[23] L. A. Zadeh, “A Theory of Approximate Reasoning," In: J. E. Hayes, D. Michie and L. I. Mikulich, Eds., Machine Intelligence, Vol. 9, Elsevier, New York, 1979, pp. 149194. 